\title{
Surveillance de l'innocuité des vaccins au Canada : déclarations au SCSESSI, 2013 à 2016
}

\author{
N Ahmadipour ${ }^{1}$, K Watkins ${ }^{1 \star}$, M Fréchette ${ }^{1}$, C Coulby $^{1}$, H Anyoti ${ }^{1}, \mathrm{~K}$ Johnson ${ }^{1}$
}

\section{Résumé}

Contexte : Le Canada dispose d'un des systèmes de surveillance de l'innocuité des vaccins les plus performants au monde. Ce système comporte un volet de surveillance passive de tous les vaccins administrés et un volet de surveillance active de tous les vaccins destinés aux enfants.

Objectifs : Fournir 1) une analyse descriptive des déclarations d'effets secondaires suivant l'immunisation (ESSI) des vaccins administrés au Canada, 2) une analyse des effets secondaires graves (ESG) et 3) une liste des dix groupes de vaccins ayant les taux de déclaration les plus élevés.

Méthodologie : Nous avons effectué des analyses descriptives des déclarations d'ESSI reçues par l'Agence de la santé publique du Canada (ASPC) jusqu'au 14 août 2017 en lien avec les vaccins commercialisés au Canada et administrés du $1^{\text {er }}$ janvier 2013 au 31 décembre 2016. Ces analyses comportaient les éléments de données suivants : type de programme de surveillance, ESSI, caractéristiques démographiques, utilisation des soins de santé, issue clinique, gravité des effets secondaires et type de vaccin.

Résultats : Au cours de la période de quatre ans, 11079 déclarations d'ESSI ont été reçues, de partout au Canada. Le taux moyen annuel de déclarations d'ESSI était de 13,4 pour 100000 doses distribuées au Canada des vaccins administrés de 2013 à 2016; ce taux était inversement proportionnel à l'âge. La majorité des déclarations (92\%) portait sur des effets non graves, soit des éruptions cutanées au point d'injection et des réactions allergiques. Dans l'ensemble, il y a eu 892 déclarations d'ESG, soit un taux de déclarations de 1,1 pour 100000 doses distribuées de 2013 à 2016. Parmi les déclarations d'ESG, les principaux ESSI les plus courants étaient l'anaphylaxie suivie des convulsions. Les vaccins conjugués contre le méningocoque du groupe $C$ (administrés de façon concomitante) étaient associés au taux d'ESSI le plus élevé, soit 91,6 pour 100000 doses distribuées. On n'a observé aucun problème inattendu d'innocuité des vaccins ni aucune augmentation de la fréquence ou de la gravité des effets secondaires attendus.

Conclusion : De 2013 à 2016, la surveillance continue de l'innocuité des vaccins commercialisés au Canada n'a mis en évidence aucune augmentation de la fréquence ou de la gravité des ESSI, ni aucun ESSI antérieurement inconnu, ni aucun secteur où des études ou des enquêtes plus approfondies s'avéraient nécessaires. Les vaccins commercialisés au Canada continuent d'avoir un excellent profil d'innocuité.

\section{Affiliation}

${ }^{1}$ Système canadien de surveillance des effets secondaires suivant l'immunisation (SCSESSI), Centre de l'immunisation et des maladies respiratoires infectieuses, Agence de la santé publique du Canada, Ottawa (Ontario)

*Correspondance : phac.aefiessi.aspc@canada.ca
Citation proposée : Ahmadipour N, Watkins K, Fréchette M, Coulby C, Anyoti H, Johnson K. Surveillance de I'innocuité des vaccins au Canada : déclarations au SCSESSI, 2013 à 2016. Relevé des maladies transmissibles au Canada 2018;44(9):233-42. https://doi.org/10.14745/ccdr.v44i09a04f

Mots-clés : innocuité des vaccins, effets secondaires, immunisation, surveillance

\section{Introduction}

Les vaccins constituent la mesure de santé publique la plus rentable que l'on connaît. Malgré cela, le Canada présente un des plus faibles taux d'immunisation parmi les pays développés. Selon une étude menée en 2013 par I'UNICEF, le Canada occupait le 28 rang sur les 29 pays à revenu élevé en ce qui concerne les taux d'immunisation (1). La réticence à la vaccination est une des raisons pouvant expliquer ces faibles taux. Heureusement, selon l'Enquête de 2015 sur la couverture vaccinale nationale des enfants, cette réticence est à la baisse, $97 \%$ des parents étant d'accord avec l'affirmation que les vaccins destinés aux enfants sont sûrs et efficaces. Les préoccupations concernant les effets secondaires éventuels étaient encore courantes, mais le taux était passé de $74 \%$ à $66 \%$ en 2011 (2). 
Le système de surveillance des vaccins du Canada est considéré comme un des meilleurs au monde (3). Le Système canadien de surveillance des effets secondaires suivant l'immunisation (SCSESSI) est un système de santé publique fédéral-provincial-territorial (FPT) qui effectue la surveillance post-commercialisation de l'innocuité des vaccins. Le SCSESSI est unique, car il assure la surveillance passive et la surveillance active. Voici ses principaux objectifs : 1) surveiller continuellement l'innocuité des vaccins commercialisés au Canada; 2) détecter les hausses de fréquence ou de gravité des réactions liées aux vaccins précédemment décelées; 3 ) cerner les effets secondaires suivant l'immunisation précédemment inconnus de l'immunisation qui pourraient être liés aux vaccins; 4) déterminer les secteurs où des études ou des enquêtes plus approfondies s'avèrent nécessaires; 5) fournir de l'information opportune sur les profils de déclaration d'effets secondaires imprévus suivant l'immunisation (ESSI) visant les vaccins commercialisés au Canada pouvant éclairer les décisions relatives à l'immunisation (4).

Au Canada, les fournisseurs de soins de santé, les fabricants et les membres du public ont chacun un rôle à jouer dans la vaccinovigilance (5). Les responsables de la santé publique (aux niveaux FPT) maintiennent une surveillance étroite de l'innocuité des vaccins par l'intermédiaire du Groupe de travail sur la vaccinovigilance (GTV) du Comité canadien sur l'immunisation. Le GTV réunit des représentants de tous les programmes d'immunisation à l'échelle FPT, ainsi que des organismes de réglementation de Santé Canada et du programme canadien de surveillance active de l'immunisation (IMPACT). Les données sur les ESSI issues de la surveillance passive sont soumises à une analyse continue par le GTVV visant à déceler les éventuels problèmes d'innocuité des vaccins pour relever et communiquer rapidement les enjeux émergents de sécurité et ainsi permettre une intervention efficace en matière de santé publique. Le présent rapport a été rédigé grâce à la contribution et au soutien des membres du GTVV.

Une description plus exhaustive des rôles et responsabilités de la pharmacovigilance post-commercialisation figure dans le Guide canadien d'immunisation et sur la page Web du SCSESSI $(4,5)$. Des renseignements sur les calendriers de vaccination des provinces et des territoires figurent sur le site Web de l'Agence de la santé publique du Canada (6).

Des rapports nationaux sur les données de surveillance de I'innocuité des vaccins sont publiés périodiquement (7-17). Le présent rapport a pour objectif de fournir a) une analyse descriptive des déclarations d'ESSI des vaccins administrés au Canada de 2013 à 2016, b) une analyse des effets secondaires graves (ESG) et c) une liste des dix groupes de vaccins ayant les taux de déclaration les plus élevés.

\section{Méthodologie}

\section{Définitions}

Un ESSI est défini comme toute manifestation clinique indésirable qui suit la vaccination, sans qu'elle ait nécessairement une relation de cause à effet avec l'administration du vaccin. L'effet secondaire peut être un signe, un symptôme ou une maladie définie (18).
Un ESG est défini comme tout ESSI qui entraîne le décès, qui met la vie en danger, qui nécessite une hospitalisation ou la prolongation d'un séjour à l'hôpital, qui cause une invalidité ou une incapacité persistante ou importante, ou qui engendre une anomalie congénitale (19). On peut établir un lien temporel, mais pas nécessairement une relation de cause à effet avec le vaccin.

\section{Sources de données}

Le SCSESSI est un processus collaboratif FPT qui comprend la soumission de déclarations d'ESSI découlant de la surveillance à la fois passive et active.

La surveillance passive débute à l'échelle des services de santé publique locaux et repose sur les déclarations d'ESSI par les fournisseurs de soins de santé, les sujets vaccinés ou leurs proches aidants. Les déclarations sont envoyées aux autorités sanitaires provinciales ou territoriales, qui sont responsables des mesures de santé publique à l'échelle de la population et de l'évaluation des programmes d'immunisation. La déclaration d'ESSI à l'organisme régional de santé publique est obligatoire dans huit provinces et territoires, et volontaire dans les six autres. Les déclarations sont ensuite transmises sur une base volontaire à l'Agence aux fins d'intégration au SCSESSI (20). Les autorités sanitaires provinciales et territoriales reçoivent également des déclarations des autorités fédérales qui fournissent des services de vaccination sur leur territoire de compétence (notamment la Direction générale de la santé des Premières nations et des Inuits, le Service correctionnel du Canada et la Gendarmerie royale du Canada). Toutes déclarations d'ESSI reçues par la Défense nationale et les Forces armées canadiennes sont signalées directement à l'Agence. À de rares occasions, des déclarations d'ESSI sont transmises directement à l'Agence par des médecins, des pharmaciens, des cliniques de voyage et des membres du public. Ces déclarations sont saisies dans le SCSESSI, et une copie de la déclaration et(ou) les renseignements sur le déclarant sont envoyés aux autorités sanitaires de la province ou du territoire d'origine.

Depuis janvier 2011, une modification apportée à la réglementation concernant la déclaration exige que les détenteurs d'une autorisation de mise sur le marché (AM) signalent les ESSI à Santé Canada; par conséquent, ces détenteurs ont graduellement cessé de signaler les ESSI à I'Agence. Ainsi, toutes les déclarations des détenteurs d'une AM (comptant pour 0,6\% de toutes les déclarations d'ESSI reçues par l'Agence) ont été exclues du présent rapport.

Depuis 1991, la surveillance active est assurée dans le cadre du programme IMPACT. Le programme IMPACT est un réseau pédiatrique hospitalier financé par l'Agence et administré par la Société canadienne de pédiatrie (21). Ce réseau compte actuellement 12 centres pédiatriques à travers le Canada, où des infirmières, sous la supervision de médecins spécialistes des maladies pédiatriques et(ou) infectieuses, étudient les admissions à l'hôpital pour relever les ESSI cibles, y compris les manifestations neurologiques ( $p$. ex. convulsions et syndrome de Guillain-Barré), les cas de thrombocytopénie, d'abcès ou de cellulite au point de vaccination, d'invagination intestinale (intussusception) et d'autres complications qui auraient pu apparaître après une vaccination et qui ont entraîné une hospitalisation $(22,23)$. 
Lors du processus de déclaration, les identificateurs personnels sont supprimés des déclarations d'ESSI avant l'envoi de celles-ci (sur support papier ou informatique) à l'Agence, où les données sont saisies dans le SCSESSI (24). L'étape de la saisie comporte un contrôle de la qualité pour éviter les divergences de données ainsi que repérer et faire concorder les déclarations en double. Les ESSI graves sont recensés en fonction de la définition de cas; les ESSI déclarés et les antécédents médicaux sont codés conformément à la terminologie de MedDRA (Dictionnaire médical international des activités de réglementation; version 17) (25). Les interventions médicales, y compris les médicaments concomitants, sont codées conformément au Système international de classification anatomique thérapeutique chimique. Le codage est suivi d'un examen systématique du cas par des professionnels de la santé dûment formés, qui déterminent la raison principale de la déclaration. Les définitions nationales de cas pour la classification des ESSI tirées du Guide de l'utilisateur du SCSESSI ont été utilisées aux fins de l'analyse médicale des cas (24).

Le nombre et le taux d'ESSI par année, la principale raison de la déclaration, la distribution selon l'âge et le sexe, les issues cliniques, une analyse de tous les ESG, ainsi qu'une liste des dix groupes de vaccins ayant les taux de déclaration les plus élevés étaient les éléments de données de l'analyse. Les résultats du présent rapport sont présentés selon l'année d'administration des vaccins (2013 à 2016).

\section{Analyse des données}

Toutes les déclarations d'ESSI transmises au SCSESSI jusqu'au 14 août 2017 dont la date de vaccination était comprise entre le $1^{\text {er }}$ janvier 2013 et le 31 décembre 2016 ont été incluses dans le présent rapport. Les données d'une juridiction n'ont pas été incluses dans cette analyse en raison de problèmes techniques de transmission et de réception des données au SCSESSI. Comme ces données n'ont pas été incluses dans le numérateur, la population de cette juridiction n'a pas été retenue dans le dénominateur lors du calcul du taux national pour 100000 habitants.

Des analyses descriptives ont été effectuées à l'aide du logiciel SAS Enterprise Guide, version 5.1 (26). Dans la mesure du possible, les taux de déclaration ont été calculés à partir des données sur les doses distribuées fournies par les détenteurs d'une autorisation de mise sur le marché, en vertu d'une entente conclue avec l'Agence. Le nombre de doses distribuées a servi de mesure substitutive pour le nombre de personnes vaccinées dans le calcul des taux pour l'ensemble des vaccins et pour chaque vaccin. Les estimations annuelles de la population de Statistique Canada ont servi de dénominateur pour le calcul des taux lorsqu'un taux ne pouvait pas être calculé à partir des doses distribuées (27).

\section{Résultats}

Au total, 11080 déclarations d'ESSI (2 750 déclarations d'ESSI en 2013, 2848 en 2014, 2845 en 2015 et 2637 en 2016) de 12 provinces et territoires ont été reçues par le SCSESSI de 2013 à 2016. Plus de 80 millions de doses de vaccins ont été distribuées, ce qui représente un taux de déclaration de 12,1 à 14,3 pour 100000 doses distribuées (figure 1).
Figure $1:$ Nombre total de déclarations d'effets secondaires suivant l'immunisation et taux de déclaration par année, 2013 à 2016

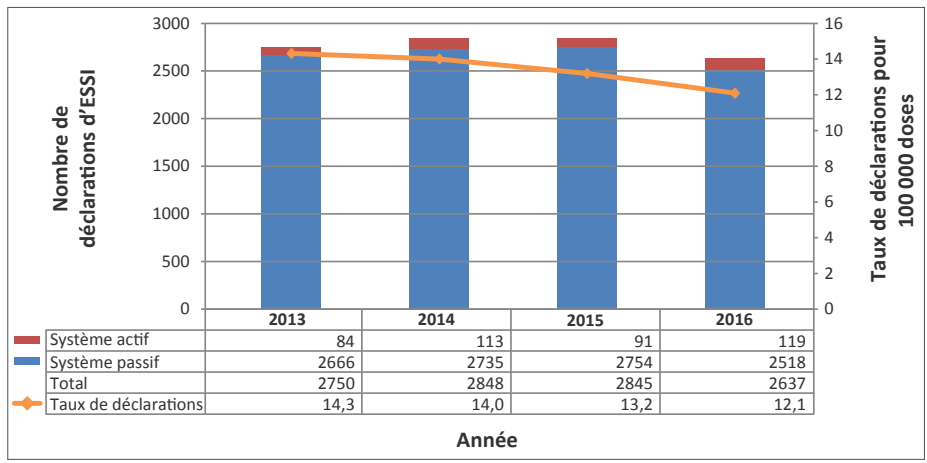

Abréviation : ESSI, effets secondaires suivant l'immunisation

\section{Distribution selon l'âge et le sexe}

Les taux de déclaration pour 100000 habitants, selon le groupe d'âge et le sexe, sont présentés à la figure 2. L'âge médian de toutes les déclarations reçues au cours de la période de déclaration était de 12 ans (plage : moins d'un mois à 104 ans). La majorité (56\%) des déclarations d'ESSI concernait des enfants et des adolescents âgés de moins de 18 ans. Le taux de déclarations le plus élevé est observé chez les nourrissons de moins d'un an (121,8 pour 100000 habitants), suivis des enfants âgés d'un à deux ans (avec un taux de 121,3 pour 100000 habitants). Des 11080 déclarations, $63 \%$ concernaient des sujets de sexe féminin. Une prédominance masculine a été observée chez les enfants de moins de sept ans et une prédominance féminine, chez les sujets de sept ans ou plus.

\section{Figure 2 : Proportion des déclarations d'effets secondaires suivant l'immunisation selon le groupe d'âge et le sexe, 2013 à 2016a}

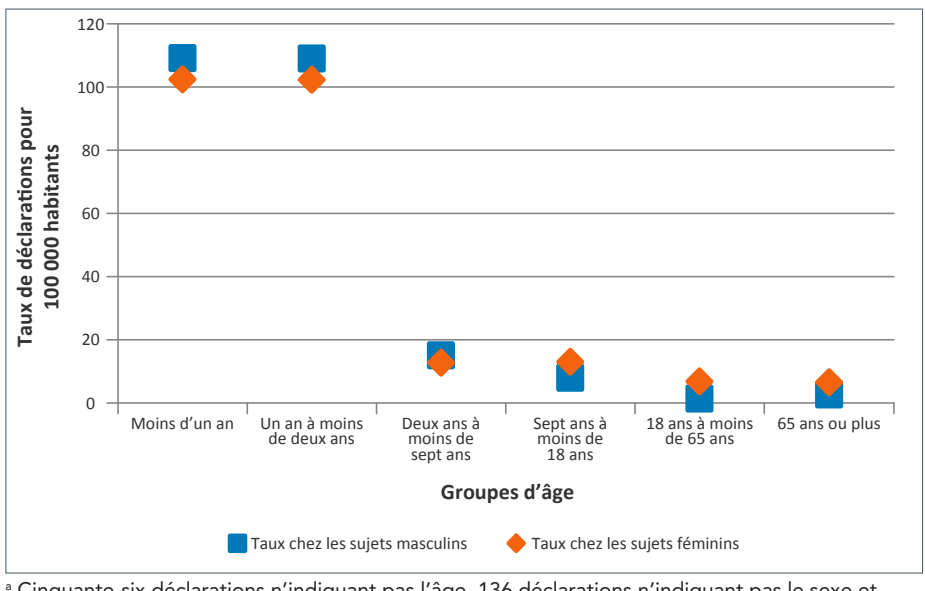

trois déclarations indiquant « autre » pour le sexe 136 déclaration

La tableau 1 présente le nombre de déclarations et les taux de déclaration pour 100000 habitants selon le groupe d'âge et l'année de vaccination. Pour toutes les années, les taux de déclaration les plus élevés ont été observés dans les groupes de sujets âgés de moins d'un an et de sujets âgés d'un an à moins de deux ans. Les taux fluctuent légèrement d'année en année dans le groupe de sujets âgés de deux à moins de sept ans; chez les sujets âgés de sept ans ou plus, les taux étaient relativement stables les quatre années. 
Tableau 1 : Nombre de déclarations d'effets secondaires suivant l'immunisation et taux de déclaration selon le groupe d'âge, 2013 à $2016^{a}$

\begin{tabular}{|c|c|c|c|c|c|}
\hline \multirow{2}{*}{$\begin{array}{l}\text { Sous-population } \\
\text { groupe d'âge }\end{array}$} & \multicolumn{5}{|c|}{$\begin{array}{l}\text { Nombre de déclarations d'ESSI (taux de } \\
\text { déclarations pour } 100000 \text { habitants) }\end{array}$} \\
\hline & 2013 & 2014 & 2015 & 2016 & $\begin{array}{l}\text { Toutes } \\
\text { les } \\
\text { années }\end{array}$ \\
\hline Moins de 1 an & $\begin{array}{r}396 \\
(117,8)\end{array}$ & $\begin{array}{r}442 \\
(131,2)\end{array}$ & $\begin{array}{r}386 \\
(114,0)\end{array}$ & $\begin{array}{r}425 \\
(124,9)\end{array}$ & $\begin{array}{r}1649 \\
(121,8)\end{array}$ \\
\hline 1 an à moins de 2 ans & $\begin{array}{r}379 \\
(112,6)\end{array}$ & $\begin{array}{r}399 \\
(117,9) \\
\end{array}$ & $\begin{array}{r}422 \\
(124,7) \\
\end{array}$ & $\begin{array}{r}444 \\
(130,6) \\
\end{array}$ & $\begin{array}{r}1644 \\
(121,3)\end{array}$ \\
\hline 2 ans à moins de 7 ans & $\begin{array}{r}313 \\
(18,3)\end{array}$ & $\begin{array}{r}331 \\
(19,3)\end{array}$ & $\begin{array}{r}242 \\
(14,1)\end{array}$ & $\begin{array}{r}213 \\
(12,5)\end{array}$ & $\begin{array}{l}1099 \\
(16,0)\end{array}$ \\
\hline $\begin{array}{l}7 \text { ans à moins de } 18 \\
\text { ans }\end{array}$ & $\begin{array}{r}425 \\
(11,5)\end{array}$ & $\begin{array}{r}436 \\
(11,8)\end{array}$ & $\begin{array}{r}453 \\
(12,2)\end{array}$ & $\begin{array}{r}458 \\
(12,2)\end{array}$ & $\begin{array}{l}1772 \\
(11,9)\end{array}$ \\
\hline $\begin{array}{l}18 \text { ans à moins de } \\
65 \text { ans }\end{array}$ & $\begin{array}{r}944 \\
(4,8)\end{array}$ & $\begin{array}{r}1006 \\
(5,0)\end{array}$ & $\begin{array}{r}1028 \\
(5,1)\end{array}$ & $\begin{array}{r}802 \\
(4,0)\end{array}$ & $\begin{array}{r}3780 \\
(4,7)\end{array}$ \\
\hline 65 ans ou plus & $\begin{array}{r}279 \\
(6,0)\end{array}$ & $\begin{array}{r}225 \\
(4,7)\end{array}$ & $\begin{array}{r}306 \\
(6,2)\end{array}$ & $\begin{array}{r}270 \\
(5,3)\end{array}$ & $\begin{array}{r}1080 \\
(5,5)\end{array}$ \\
\hline Tous les âges & $\begin{array}{r}2736 \\
(9,0)\end{array}$ & $\begin{array}{r}2839 \\
(9,2)\end{array}$ & $\begin{array}{r}2837 \\
(9,1)\end{array}$ & $\begin{array}{r}2612 \\
(8,3)\end{array}$ & $\begin{array}{r}11024 \\
(8,9)\end{array}$ \\
\hline
\end{tabular}

Abréviation : ESSI, effets secondaires suivant l'immunisation

a Cinquante-six déclarations n'indiquant pas l'âge ont été exclues

\section{Principale raison de la déclaration}

Lors de l'analyse médicale du cas, la principale raison de la déclaration a été classée dans une catégorie générale d'ESSI, puis dans une sous-catégorie plus précise. Le tableau 2 liste les catégories générales des ESSI et leurs sous-catégories, selon le nombre total de déclarations. Les principales raisons les plus courantes des déclarations étaient les réactions au niveau du site de vaccination suivies des éruptions cutanées seulement, qui comptaient pour $54 \%$ de l'ensemble des déclarations transmises (8\% de toutes les déclarations d'ESG) de 2013 à 2016.

Tableau 2 : Fréquence des effets secondaires et pourcentage d'effets secondaires graves pour chaque sous-catégorie d'effets secondaires suivant I'immunisation, 2013 à 2016

\begin{tabular}{|c|c|c|c|}
\hline ESSI général & $\begin{array}{l}\text { Sous-catégorie de } \\
\text { I'ESSI général }\end{array}$ & $\begin{array}{l}\text { Nombre de } \\
\text { déclarations } \\
(\mathrm{N}=11080)\end{array}$ & $\begin{array}{l}\text { Grave } \\
(\%)\end{array}$ \\
\hline \multirow{3}{*}{$\begin{array}{l}\text { Réactions } \\
\text { allergiques } \\
\text { et autres } \\
\text { manifestations } \\
\text { allergiques }\end{array}$} & Anaphylaxie & 111 & 100 \\
\hline & $\begin{array}{l}\text { Autres manifestations } \\
\text { allergiques }^{\mathrm{a}}\end{array}$ & 1526 & 1 \\
\hline & $\begin{array}{l}\text { Syndrome } \\
\text { oculo-respiratoire }\end{array}$ & 158 & 1 \\
\hline \multirow{6}{*}{$\begin{array}{l}\text { Infection/ } \\
\text { syndrome/ } \\
\text { symptômes } \\
\text { systémiques (ISS) }\end{array}$} & Fièvre seulement & 52 & 21 \\
\hline & Infection & 182 & 34 \\
\hline & Syndrome grippal & 82 & 4 \\
\hline & $\begin{array}{l}\text { Éruption cutanée } \\
\text { accompagnée de fièvre } \\
\text { et(ou) d'une autre } \\
\text { affection }\end{array}$ & 364 & 5 \\
\hline & $\begin{array}{l}\text { Syndrome indiqué dans } \\
\text { les déclarations d'ESSI } \\
\text { (p. ex. Kawasaki) }\end{array}$ & 90 & 79 \\
\hline & $\begin{array}{l}\text { Systémique (plusieurs } \\
\text { systèmes de l'organisme } \\
\text { sont touchés) }\end{array}$ & 389 & 14 \\
\hline
\end{tabular}

Page 236

RMTC • le 6 septembre $2018 \bullet$ Volume 44-9
Tableau 2 (suite) : Fréquence des effets secondaires et pourcentage d'effets secondaires graves pour chaque sous-catégorie d'effets secondaires suivant I'immunisation, 2013 à 2016

\begin{tabular}{|c|c|c|c|}
\hline ESSI général & $\begin{array}{l}\text { Sous-catégorie de } \\
\text { I'ESSI général }\end{array}$ & $\begin{array}{l}\text { Nombre de } \\
\text { déclarations } \\
(\mathrm{N}=11080)\end{array}$ & $\begin{array}{l}\text { Grave } \\
(\%)\end{array}$ \\
\hline \multirow{8}{*}{$\begin{array}{l}\text { Manifestations } \\
\text { neurologiques }\end{array}$} & Méningite aseptique & 16 & 81 \\
\hline & Ataxie/cérébellite ${ }^{b}$ & 9 & 67 \\
\hline & Paralysie de Bell & 29 & 0 \\
\hline & $\begin{array}{l}\text { Encéphalite/ } \\
\text { encéphalomyélite aiguë } \\
\text { disséminée (EMAD)/myélite }\end{array}$ & 25 & 87 \\
\hline & Syndrome de Guillain-Barré & 32 & 88 \\
\hline & $\begin{array}{l}\text { Autre paralysie durant plus } \\
\text { de } 1 \text { jour }\end{array}$ & 7 & 43 \\
\hline & Convulsions & 389 & 48 \\
\hline & $\begin{array}{l}\text { Autre manifestation } \\
\text { neurologique }\end{array}$ & 94 & 20 \\
\hline \multirow{3}{*}{$\begin{array}{l}\text { Éruption cutanée } \\
\text { seulement }\end{array}$} & Généralisée & 1493 & 0 \\
\hline & Localisée & 225 & 0 \\
\hline & $\begin{array}{l}\text { Endroit non précisé/ } \\
\text { étendue inconnue }\end{array}$ & 122 & 0 \\
\hline \multirow{3}{*}{$\begin{array}{l}\text { Anxiété due à } \\
\text { l'immunisation }\end{array}$} & Présyncope & 31 & 3 \\
\hline & Syncope & 57 & 2 \\
\hline & $\begin{array}{l}\text { Autre manifestation } \\
\text { d'anxiétéd }\end{array}$ & 33 & 6 \\
\hline \multirow{5}{*}{$\begin{array}{l}\text { Réactions au } \\
\text { niveau du site de } \\
\text { vaccination }\end{array}$} & Abcès (infecté ou stérile) & 54 & 11 \\
\hline & Cellulite & 907 & 4 \\
\hline & $\begin{array}{l}\text { Enflure importante à un } \\
\text { membre }\end{array}$ & 363 & 1 \\
\hline & $\begin{array}{l}\text { Douleur au membre vacciné } \\
\text { durant } 7 \text { jours ou plus }\end{array}$ & 134 & 1 \\
\hline & Autre réaction locale ${ }^{f}$ & 2691 & 1 \\
\hline $\begin{array}{l}\text { Erreur de } \\
\text { vaccination }\end{array}$ & Erreur de vaccination & 9 & 0 \\
\hline \multirow{12}{*}{$\begin{array}{l}\text { Autres } \\
\text { manifestations }\end{array}$} & Arthralgie & 73 & 5 \\
\hline & Arthrite & 36 & 28 \\
\hline & $\begin{array}{l}\text { Manifestation gastro- } \\
\text { intestinale }\end{array}$ & 549 & 3 \\
\hline & $\begin{array}{l}\text { Épisode hypotonique- } \\
\text { hyporéactif }\end{array}$ & 74 & 26 \\
\hline & $\begin{array}{l}\text { Invagination intestinale } \\
\text { (intussusception) }\end{array}$ & 29 & 83 \\
\hline & Anesthésie/paresthésie & 203 & 2 \\
\hline & Parotidite & 9 & 0 \\
\hline & Pleurs persistants & 72 & 3 \\
\hline & $\begin{array}{l}\text { Syndrome de mort subite } \\
\text { du nourrisson }\end{array}$ & 6 & 100 \\
\hline & Mort subite & 3 & 100 \\
\hline & Thrombocytopénie & 43 & 81 \\
\hline & Autres manifestations ${ }^{h}$ & 327 & 14 \\
\hline
\end{tabular}

Abréviation : ESSI, effets secondaires suivant l'immunisation

a La sous-catégorie "Autres » comprend, sans s'y limiter, I'hypersensibilité et l'urticaire

b L'ataxie cérébelleuse est définie par l'apparition soudaine d'une ataxie tronculaire et de troubles de la démarche (22). Notons que l'on suppose ici une absence de signes cérébelleux accompagnés d'autres signes d'encéphalite ou d'encéphalomyélite aiguë disséminée (EMAD); dans ce cas, la manifestation serait classée en fonction de la définition de cas de la Brighton Collaboration (23) "La sous-catégorie "Autres " comprend, sans s'y limiter, les phénomènes convulsifs et les migraines 'La sous-catégorie « Autres " comprend, sans s'y limiter, les étourdissements et la dyspnée

e Une enflure importante d'un segment complet d'un membre proximal et(ou) distal, un "segment " étant la partie comprise entre deux articulations (24)

La sous-catégorie « Autres » comprend, sans s'y limiter, la douleur au site de vaccination et l'enflure au site de vaccination

" Autres " : autres manifestations décrites dans le formulaire de déclaration du Système canadien de surveillance des effets secondaires suivant l'immunisation (SCSESSI).

h La sous-catégorie " Autres » comprend, sans s'y limiter, l'adénopathie et l'arthralgie 
La figure 3 présente la répartition des ESSI selon la principale raison et le groupe d'âge indiqués dans la déclaration,

déterminés lors de l'analyse médicale du cas. Les réactions au niveau du site de vaccination étaient les effets les plus courants, suivies des éruptions cutanées et des réactions allergiques. Les réactions au niveau du site de vaccination représentaient la majorité des effets dans tous les groupes d'âge, sauf chez les enfants de moins de deux ans. Chez les enfants de moins d'un an, les ESSI les plus souvent signalés étaient classés sous " autres » (ce qui comprenait les sous-catégories telles que les troubles gastro-intestinaux, les pleurs persistants et les épisodes hypotoniques-hyporéactifs), suivis des éruptions cutanées. Chez les enfants âgés d'un an à moins de deux ans, I'ESSI le plus souvent signalé était l'éruption cutanée, suivie des réactions au niveau du site de vaccination et des infections/syndromes/ symptômes systémiques (ISS).

Figure 3 : Proportion des principaux effets secondaires suivant l'immunisation déclarés par groupe d'âge, 2013 à $2016^{a}$

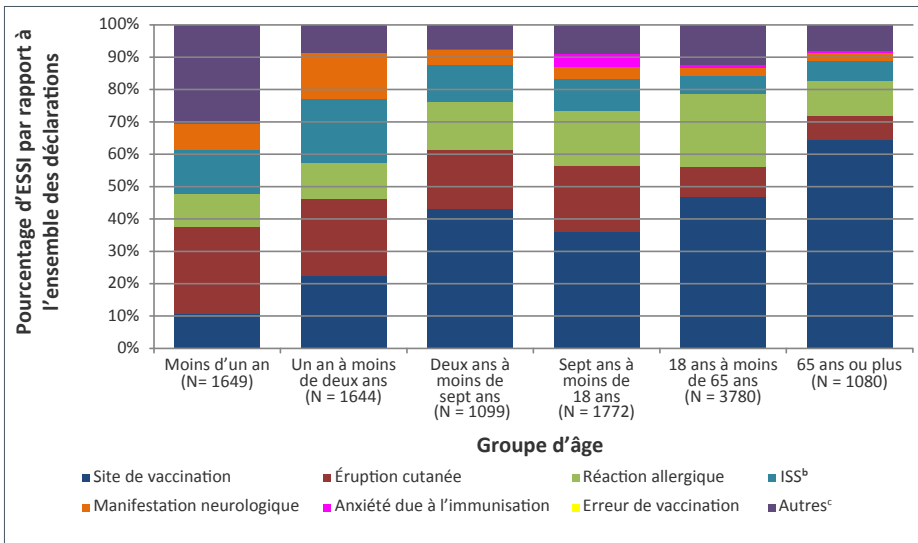

Abréviations : ESSI, effets secondaires suivant l'immunisation; ISS, infection/syndrome/symptômes systémiques; $\mathrm{N}$, nombre

a Cinquante-six déclarations n'indiquant pas l'âge sont exclues de cette figure

b Les ISS sont des manifestations touchant principalement plusieurs systèmes organiques, souvent accompagnées de fièvre. Ces manifestations comprennent des sous-catégories comme des syndromes reconnus ( $p$. ex. le syndrome de Kawasaki, la fibromyalgie), la fièvre seule, le syndrome grippal et les manifestations systémiques (comme la fatigue, le malaise et la léthargie). Elles englobent également les signes d'infection d'une ou de plusieurs parties du corps

'Les autres manifestations comprennent l'arthralgie, l'arthrite, l'épisode hypotonique-hyporéactif, I'invagination intestinale (intussusception), les maladies gastro-intestinales, l'anesthésie ou la paresthésie, la parotidite, les pleurs persistants, la thrombocytopénie, le syndrome de mort subite du nourrisson (SMSN) et la mort subite

La figure 4 présente les catégories générales d'ESSI et la proportion dans chaque catégorie où des effets ont été considérés comme graves. La proportion variait de 0 à $46 \%$. La proportion d'effets graves était la plus élevée dans la catégorie des manifestations neurologiques (46\%), suivie par celle des ISS (19\%). Notons que les erreurs de vaccination n'apparaissaient que dans un petit nombre de déclarations (neuf déclarations $\left.d^{\prime} E S S I\right)$ et dans aucune déclaration d'ESSI grave.

Dans le cas des sujets de moins de 18 ans, $7 \%(n=407)$ de l'ensemble des déclarations d'ESSI provenaient de la surveillance active. Bien que cette proportion soit petite, elle représente $56 \%(n=401)$ de toutes les déclarations d'ESSI graves soumises pour ce groupe d'âge, ce qui illustre la contribution du système de surveillance active dans le milieu hospitalier. (Note : données non présentées; les valeurs ne correspondent pas aux pourcentages car ces derniers ont été arrondis au nombre entier le plus près.)
Figure 4 : Principaux effets secondaires suivant l'immunisation selon la gravité, 2013 à 2016

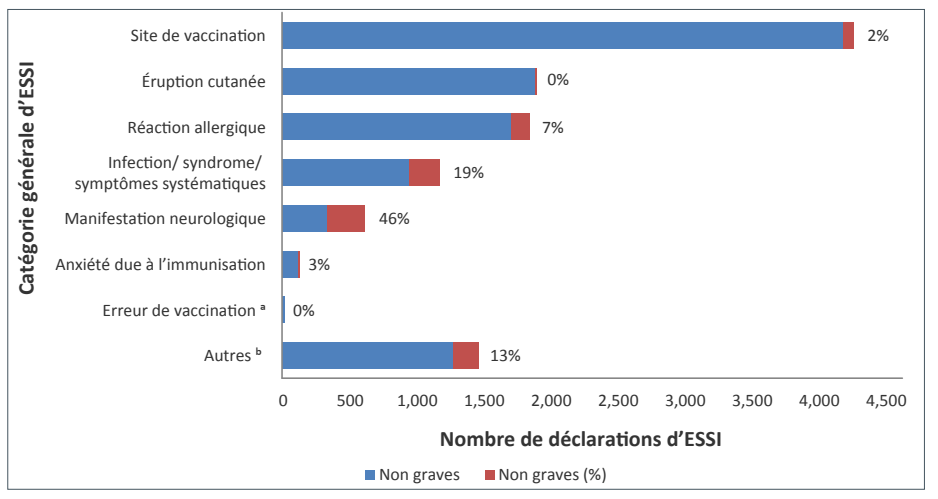

Abréviation : ESSI, effets secondaires suivant l'immunisation

Les erreurs de vaccination n'apparaissaient que dans un petit nombre de déclarations (neuf déclarations d'ESSI) et dans aucune déclaration d'ESSI grave

b Les autres manifestations comprennent l'arthralgie, I'arthrite, l'épisode hypotonique-hyporéactif, I'invagination intestinale (intussusception), les maladies gastro-intestinales, l'anesthésie ou la paresthésie, la parotidite, les pleurs persistants, la thrombocytopénie, le syndrome de la mort subite du nourrisson et le syndrome de la mort subite

\section{Utilisation des soins de santé}

Le tableau 3 présente le plus haut niveau de soins nécessaire suivant la survenue d'un ESSI. L'utilisation la plus souvent signalée des soins de santé était la visite non urgente dans un établissement de soins (37\%). La plupart des personnes faisant l'objet d'une déclaration d'ESSI (93\%) n'ont pas eu besoin d'être hospitalisées. Dans près de $25 \%$ des cas, aucun soin de santé n'a été demandé.

\section{Tableau 3 : Utilisation des soins de santé en raison d'effets secondaires suivant l'immunisation, 2013 à 2016}

\begin{tabular}{|c|c|c|}
\hline $\begin{array}{l}\text { Plus haut niveau de soins } \\
\text { recouru }\end{array}$ & Nombre & $\%$ \\
\hline $\begin{array}{l}\text { Hospitalisation requise } \\
\text { ( } 24 \mathrm{~h} \text { ou plus) }\end{array}$ & 764 & 7 \\
\hline Prolongation du séjour à l'hôpital & 4 & Moins de 1 \\
\hline Visite au service d'urgence & 2126 & 19 \\
\hline Visite non urgente & 4084 & 37 \\
\hline $\begin{array}{l}\text { Obtention de l'avis d'un professionnel de } \\
\text { la santé par téléphone }\end{array}$ & 487 & 4 \\
\hline Aucun & 2542 & 23 \\
\hline Inconnu & 323 & 3 \\
\hline Donnée manquante & 750 & 7 \\
\hline Total & 11080 & 100 \\
\hline
\end{tabular}

\section{Résultat}

L'issue clinique au moment de la déclaration pour l'ensemble de ESSI figure au tableau 4. La guérison complète a été signalée dans $76 \%$ des déclarations. Dans les cas où la guérison est incomplète au moment de la déclaration (18\%), cette dernière est révisée lorsque l'information est envoyée au SCSESSI. 
Tableau 4 : Issue clinique au moment de la déclaration dans tous les cas, 2013 à 2016

\begin{tabular}{|l|r|r|}
\hline \multicolumn{1}{|c|}{ Résultat } & \multicolumn{1}{c|}{ Nombre } & \multicolumn{1}{c|}{$\%$} \\
\hline Guérison complète & 8464 & 76 \\
\hline $\begin{array}{l}\text { Guérison non obtenue au moment de } \\
\text { la déclaration }\end{array}$ & 1948 \\
\hline Invalidité/incapacité permanente & 12 & Moins de 1 \\
\hline Décès & 32 & Moins de 1 \\
\hline Inconnu & 532 & 5 \\
\hline Donnée manquante & 11080 & Moins de 1 \\
\hline Total & & 100 \\
\hline
\end{tabular}

\section{Déclaration d'effets secondaires graves}

Dans l'ensemble, il y a eu 892 déclarations d'ESG sur les plus de 80 millions de doses de vaccin distribuées au cours de la période de déclaration. Cela représente un taux de 1,1 pour 100000 doses distribuées et $8 \%$ de toutes les déclarations d'ESSI durant la période de quatre ans (distribution : de 1,0 à 1,2 déclaration pour 100000 doses distribuées). La figure 5 présente la proportion de déclarations d'ESG découlant des hospitalisations ( $n=745)$, des événements qui mettent la vie en danger $(n=103)$, des décès $(n=32)$, des invalidités résiduelles $(n=11)$ et d'autres raisons $(n=1)$.

\section{Figure 5 : Classification des déclarations d'effets secondaires graves, 2013 à 2016}

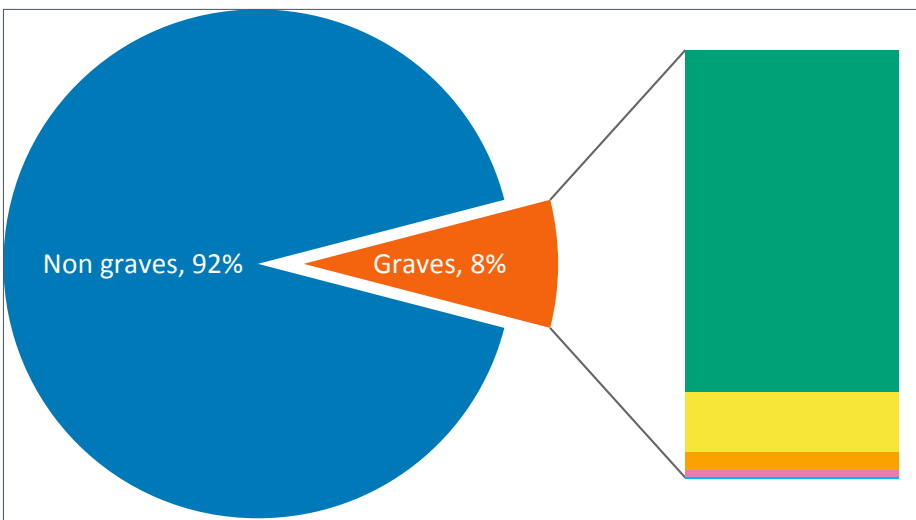

- Hospitalisation (84\%) Événement qui met la vie en danger (12\%) Décès (4\%) - Invalidité résiduelle (1\%) - Autre $(0.1 \%)$

Note : L'arrondissement des pourcentages donne un total légèrement supérieur à $100 \%$

Parmi les déclarations d'ESG, les principaux ESSI les plus souvent signalés étaient les convulsions $(20,1 \%)$, suivies de l'anaphylaxie $(12,4 \%)$. La majorité des ESG a été observée chez les enfants et les adolescents de moins de 18 ans (80\%). Plus de la moitié de ces ESG touchaient les enfants de moins de deux ans; c'est un résultat auquel on peut s'attendre, en raison du nombre de vaccins administrés aux enfants de ce groupe d'âge pour les protéger au moment où ils sont le plus vulnérables aux maladies évitables par la vaccination.

Dans la majorité des cas d'ESG (73\%), la guérison était complète au moment de la déclaration. Dans environ $15 \%(n=137)$ des déclarations d'ESG, les patients n'étaient pas complètement rétablis au moment de la déclaration. Ces déclarations sont révisées lorsque le SCSESSI reçoit des renseignements actualisés. Les autres issues faisant l'objet de déclarations d'ESG comprenaient le décès $(n=32 ; 3,6 \%)$, l'invalidité/incapacité permanente $(n=10 ; 1,1 \%)$, l'issue inconnue $(n=60 ; 6,7 \%)$ et les données manquantes concernant l'issue $(n=2 ; 0,3 \%)$.

Les 32 déclarations de décès ont été rigoureusement examinées, et on a jugé que ces décès n'étaient pas attribuables aux vaccins administrés. Neuf de ces décès $(28 \%)$ ont été déclarés dans le plus jeune groupe d'âge (moins d'un an); six étaient attribuables au syndrome de mort subite du nourrisson et trois découlaient d'un état pathologique sous-jacent (soit un infarctus cérébral, un arrêt cardiaque et des complications survenues pendant l'alimentation par sonde naso-gastrique). Sept décès ont été déclarés dans le groupe des enfants âgés d'un à moins de deux ans, dont trois associés à une mort subite, trois causés par une infection non liée au vaccin administré (infection à pneumocoques, infection à Streptococcus pneumoniae/à staphylocoques, encéphalite nécrosante) et un attribuable à un problème préexistant (lésion cérébrale). Deux décès attribuables à des états pathologiques sous-jacents (maladie congénitale et lésion cérébrale grave subie à la naissance) ont été déclarés dans le groupe des enfants âgés de deux à moins de sept ans et un décès causé par une affection préexistante (épilepsie), dans le groupe des sujets âgés de sept à moins de 18 ans. Les 13 autres décès concernent des adultes, soit six dans le groupe des 18 à 65 ans et sept dans le groupe des personnes âgées de 65 ans ou plus (distribution : 49-93 ans); toutes ces personnes présentaient un état pathologique préexistant. Les causes de décès énumérées comprenaient les maladies cardiovasculaires (infarctus du myocarde, cardiopathie ischémique et athérosclérose), les pneumopathies (maladie pulmonaire obstructive chronique, asthme), les affections du système nerveux central (démence, encéphalite associée à $\mathrm{H} 1 \mathrm{~N} 1$, paralysie cérébrale et empyème intracrânien), les tumeurs malignes (cancer du poumon et du sein), l'immunodépression et le diabète de type 2 .

\section{Dix groupes de vaccins associés aux plus hauts taux de déclarations d'ESSI}

Lors d'une visite de vaccination, un ou plusieurs vaccins peuvent être administrés. Pour l'ensemble des 11080 déclarations, 18134 vaccins avaient été administrés, soit une moyenne de deux vaccins par déclaration (distribution : de 1 à 6 ). Le tableau 5 indique les 10 groupes de vaccins associés aux taux de déclarations les plus élevés et 1 ) le nombre et le taux de déclarations d'ESSI pour chaque vaccin (administré seul ou en association avec d'autres vaccins), 2) le nombre et la proportion de déclarations en lien avec un vaccin administré seul et 3) le nombre et le taux de déclarations d'ESG associés à l'administration de ce vaccin seul. Le vaccin conjugué contre le méningocoque du groupe $C$ était associé au taux de déclarations d'ESSI le plus élevé, soit de 91,6 pour 100000 doses distribuées $(n=1346)$, dont la vaste majorité était constituée d'effets non graves. Bien que le vaccin conjugué contre le méningocoque du groupe $C$ soit associé au taux le plus élevé, le nombre le plus élevé de déclarations d'ESSI concernait le vaccin antigrippal $(n=3405 ; 7,1$ pour 100000 doses distribuées; données non présentées). 
Tableau 5 : Liste des 10 principaux groupes de vaccins pour l'ensemble des déclarations d'effets secondaires suivant l'immunisation et nombre total de déclarations et d'effets secondaires graves en lien avec le vaccin administré seul, 2013 à 2016

\begin{tabular}{|c|c|c|c|c|c|c|c|}
\hline \multirow[t]{2}{*}{$\begin{array}{l}\text { Groupe de } \\
\text { vaccins }\end{array}$} & \multirow[t]{2}{*}{$\begin{array}{l}\text { Nom } \\
\text { commercial } \\
\text { des vaccins }\end{array}$} & \multicolumn{2}{|c|}{$\begin{array}{l}\text { Taux de } \\
\text { déclara- } \\
\text { tions pour } \\
100000 \\
\text { doses } \\
\text { distribuées }\end{array}$} & \multicolumn{2}{|c|}{$\begin{array}{l}\text { Déclara- } \\
\text { tions pour } \\
\text { un vaccin } \\
\text { administré } \\
\text { seul }\end{array}$} & \multicolumn{2}{|c|}{$\begin{array}{l}\text { Déclara- } \\
\text { tions } \\
\text { d'ESG } \\
\text { pour un } \\
\text { vaccin } \\
\text { administré } \\
\text { seul }^{\mathrm{a}}\end{array}$} \\
\hline & & $\mathbf{N}$ & $\%$ & $\mathbf{N}$ & $\%$ & $\mathbf{N}$ & Taux $^{a}$ \\
\hline $\begin{array}{l}\text { Vaccins conjugués } \\
\text { contre le } \\
\text { méningocoque du } \\
\text { groupe C }\end{array}$ & $\begin{array}{l}\text { Meningitec }{ }^{\mathrm{MD}} \\
\text { Menjugate }^{\mathrm{MD}} \\
\text { NeisVac-C }^{\mathrm{MD}}\end{array}$ & 1346 & 91,6 & 33 & 2 & 4 & 0,3 \\
\hline $\begin{array}{l}\text { Diphtérie, } \\
\text { anatoxine } \\
\text { tétanique, } \\
\text { coqueluche } \\
\text { acellulaire, } \\
\text { antipolio- } \\
\text { myélitique inactivé }\end{array}$ & $\begin{array}{l}\text { QuadracelMD } \\
\text { Infanrix }^{\mathrm{MD}} \text {-IPV }\end{array}$ & 167 & 76,8 & 92 & 55 & 4 & 1,8 \\
\hline $\begin{array}{l}\text { Diphtérie, } \\
\text { anatoxine } \\
\text { tétanique, } \\
\text { coqueluche } \\
\text { acellulaire, } \\
\text { hépatite B, } \\
\text { antipolio- } \\
\text { myélitique inactivé, } \\
\text { Hæmophilus } \\
\text { influenzæ de } \\
\text { type B }\end{array}$ & $\begin{array}{l}\text { INFANRIX } \\
\text { hexamD }\end{array}$ & 462 & 65,9 & 35 & 8 & 2 & 0,3 \\
\hline $\begin{array}{l}\text { Antipneumo- } \\
\text { coccique conjugué }\end{array}$ & $\begin{array}{l}\text { Prevnar } \\
\text { Synflorix } \\
\text { Prevnar }^{\mathrm{MD}} 13\end{array}$ & 2098 & 64,4 & 64 & 3 & 5 & 0,2 \\
\hline $\begin{array}{l}\text { Rougeole, } \\
\text { oreillons, rubéole, } \\
\text { varicelle }\end{array}$ & $\begin{array}{l}\text { Priorix-TetraMD } \\
\text { ProQuad }\end{array}$ & 1075 & 59,8 & 86 & 8 & 11 & 0,6 \\
\hline $\begin{array}{l}\text { Méningocoque du } \\
\text { groupe B }\end{array}$ & Bexsero $^{\mathrm{MD}}$ & 212 & 57,1 & 160 & 75 & 17 & 4,6 \\
\hline $\begin{array}{l}\text { Vaccin conjugué } \\
\text { contre } \\
\text { Hæmophilus b }\end{array}$ & $\begin{array}{l}\text { ACT-HIB } \\
\text { Hiberix }^{\mathrm{MD}} \\
\text { suspension } \\
\text { PedvaxHib }\end{array}$ & 39 & 45,9 & 4 & 10 & 0 & 0,0 \\
\hline Rage & $\begin{array}{l}\text { IMOVAXMD }^{\text {MOV }} \\
\text { Rabies } \\
\text { RabAvert }\end{array}$ & 80 & 43,2 & 64 & 80 & 4 & 2,2 \\
\hline $\begin{array}{l}\text { Polysaccharides de } \\
\text { pneumocoques }\end{array}$ & $\begin{array}{l}\text { Pneumo }{ }^{\mathrm{MD}} 23 \\
\text { Pneumovax }^{\mathrm{MD}} \\
23\end{array}$ & 915 & 42,9 & 452 & 50 & 28 & 1,3 \\
\hline $\begin{array}{l}\text { Diphtérie, } \\
\text { anatoxine } \\
\text { tétanique, } \\
\text { coqueluche } \\
\text { acellulaire, } \\
\text { antipoliomyélitique } \\
\text { inactivé, } \\
\text { Hæmophilus } \\
\text { influenzæ de } \\
\text { type B }\end{array}$ & $\begin{array}{l}\text { PediacelMD } \\
\text { Infanrix } \\
\text { - IPV/HIB } \\
\text { Pentacel }^{\mathrm{MC}}\end{array}$ & 1512 & 40,7 & 422 & 28 & 38 & 1,0 \\
\hline
\end{tabular}

Abréviations : ESG, effet secondaire grave; $\mathrm{N}$, nombre

a Le taux de déclaration est par 100000 doses distribuées

\section{Discussion}

De 2013 à 2016, le taux annuel moyen de déclarations d'ESSI était de 13,4 pour 100000 doses distribuées (distribution : de 12,1 à 14,3) ou de 8,9 pour 100000 habitants. Ce taux est inférieur à celui signalé dans le rapport annuel de 2012 du SCSESSI, qui était de 10,1 pour 100000 habitants (17) et à celui du rapport annuel australien de 2015, qui était de 12,3 pour 100000 habitants (28). Selon les estimations, les données manquantes d'une région auraient compté pour 2000 déclarations d'ESSI au cours des quatre années; nous avons donc recalculé le taux pour 100000 habitants, et les taux globaux étaient encore inférieurs à ceux de 2012. Les différences quant aux taux de déclarations canadiens peuvent être attribuables à une sous-déclaration, à l'utilisation de vaccins combinés chez les enfants, ce qui pourrait se traduire par un nombre moins élevé de déclarations ( $p$. ex. les vaccins contre la rougeole, la rubéole et les oreillons [RRO] et les vaccins contre la varicelle ont été combinés en un vaccin RROV), à des variations dans les déclarations de manifestations légères attendues, ainsi qu'à l'exclusion des déclarations des détenteurs d'une AM de la présente analyse. En outre, on peut s'attendre à ce que les structures de déclaration soient différentes en Australie. Aucun problème d'innocuité des vaccins ni aucune hausse de la fréquence ou de la gravité des effets secondaires attendus n'ont été observés au cours de la période de déclaration.

La majorité des déclarations d'ESSI concernait des vaccins administrés à des nourrissons et à de jeunes enfants. De tels résultats étaient prévisibles, étant donné que ce groupe d'âge reçoit de nombreux vaccins - lors d'une seule visite et à de courts intervalles -, ce qui offre aux fournisseurs de soins de santé plusieurs occasions de faire des déclarations. Une plus grande proportion (63\%) des déclarations concernait des sujets féminins. Ce résultat est comparable à d'autres constats, révélant que les femmes de la population adulte signalent invariablement plus d'effets secondaires $(7-17,29)$. Les différences observées entre les sexes en fonction de l'âge peuvent aussi être en partie expliquées par une plus importante couverture vaccinale chez les femmes (30). Les différences propres au sexe étaient significatives $(p<0,05)$ chez les sujets de sept ans et plus, le taux de déclarations d'ESSI observé chez les sujets féminins étant supérieur à celui des sujets masculins. Ces différences cadrent avec celles d'autres études portant sur les différences quant aux taux de déclarations d'ESSI propres au sexe $(29,31,32)$. Parmi les enfants âgés de sept ans ou moins, on comptait plus de déclarations d'ESSI chez les sujets masculins que chez les sujets féminins; cependant, cette différence n'était pas significative.

La majorité des effets secondaires déclarés à la suite de l'administration d'environ 80 millions de doses de vaccins distribuées au Canada était constituée de réactions non graves et attendues au niveau du site de vaccination, comme la douleur et la rougeur, l'éruption cutanée, ainsi que de réactions allergiques, comme l'hypersensibilité. Au cours de la période de quatre ans, $8 \%$ des ESSI déclarés étaient des effets secondaires graves. Cette proportion est légèrement plus élevée que celle signalée aux États-Unis durant la même période $(5 \%)$ et comparable à celle des années antérieures au Canada, mais est inférieure à celle observée en Australie en 2015 (15\%) (17,28,33). La majorité des ESG touchaient les enfants et les adolescents, ce qui peut en 
partie s'expliquer par le programme IMPACT, qui représente plus de la moitié de l'ensemble des déclarations d'ESSI relatives aux sujets de moins de 18 ans et qui cible des effets particuliers chez les enfants $(20,34)$. Au moment de la déclaration, la majorité des cas d'ESG étaient complètement guéris. On a jugé qu'aucun des 32 décès déclarés au cours de la période de quatre ans n'était attribuable aux vaccins administrés.

\section{Limites}

La surveillance passive des ESSI est sujette à certaines limites, comme la sous-déclaration, l'incertitude quant à la validité du diagnostic d'un effet signalé, le manque de renseignements concernant d'autres causes possibles, comme un état pathologique sous-jacent ou des médicaments concomitants, ainsi que les différentes pratiques en matière de déclaration d'une région à l'autre du Canada, qui pourraient entraîner une surdéclaration ou une sous-déclaration des ESSI légers dans certaines provinces et certains territoires. Malgré ces limites, la surveillance passive est utile pour détecter d'éventuels signalements relatifs aux vaccins, qui peuvent alors faire l'objet d'enquête et être vérifiés. La saisonnalité n'a pas été analysée en tant que variable potentielle dans le présent rapport.

La surveillance active a aussi des limites. Le programme IMPACT cible des ESSI préétablis (comme les convulsions), ce qui peut limiter la capacité de ce programme de repérer de nouvelles réactions indésirables aux vaccins. En outre, le programme IMPACT est axé sur les cas d'enfants hospitalisés, ce qui signifie que seuls les cas les plus graves sont détectés. Enfin, le programme IMPACT n'est pas exhaustif, car il ne couvre que $90 \%$ des lits et des hospitalisations pédiatriques en soins tertiaires au Canada $(23,34)$. Malgré ces limites, ce programme peut jouer un rôle important dans la surveillance de l'innocuité des vaccins, en repérant de façon active des ESSI graves ciblés dans la population pédiatrique.

De plus, il est impossible de déterminer le nombre de doses administrées dans la population; par conséquent, on se sert soit des doses distribuées, soit des statistiques de la population comme dénominateur. L'utilisation des doses distribuées peut entraîner une sous-estimation des taux, car on ne tient pas compte des pertes. En outre, les doses distribuées au cours d'une année peuvent ne pas être administrées cette même année, ce qui réduit d'autant plus l'exactitude du dénominateur des doses distribuées. Malgré ces limites, un dénominateur fondé sur les doses distribuées a servi au calcul des taux dans le présent rapport, car un dénominateur fondé sur la population suppose une distribution similaire des doses de vaccin dans les sous-groupes de la population, même si ce n'est peut-être pas systématiquement le cas.

\section{Conclusion}

Le Canada dispose d'un système de surveillance des vaccins exhaustif qui a permis de relever un taux moyen de déclaration d'ESSI de 8,9 pour 100000 habitants. On n'a observé aucun problème inattendu d'innocuité des vaccins ni aucune augmentation de la fréquence ou de la gravité des effets secondaires attendus. La majorité des ESSI déclarés était attendue et de nature bénigne; on n'a observé aucun effet secondaire grave ni aucune hausse du taux d'effets secondaires graves inattendus. Les vaccins commercialisés au Canada continuent d'avoir un excellent profil d'innocuité.

\section{Déclaration des auteurs}

N. A. - Conceptualisation, méthodologie, validation, rédaction ébauche initiale

K. W. - Rédaction - examen et révision, supervision

M. F. - Conceptualisation, méthodologie, validation, logiciel, analyse officielle, rédaction - ébauche initiale, rédaction examen et révision

C. C. - Logiciel, analyse officielle, validation, rédaction - ébauche initiale, rédaction - examen et révision

H. A. - Validation, rédaction - examen et révision, supervision

K. J. - Rédaction - examen et révision

\section{Conflit d'intérêts}

Aucun.

\section{Remerciements}

La production du présent rapport ne serait pas possible sans la contribution du public, des professionnels de la santé publique et des autorités sanitaires locales, régionales, provinciales et territoriales, qui ont transmis des déclarations au SCSESSI ni sans la collaboration continue des membres du Groupe de travail sur la vaccinovigilance. Nous tenons également à remercier les membres de ce groupe pour leurs commentaires et leur soutien tout au long de l'élaboration de ce rapport. Nous remercions chaque personne qui prend le temps de soumettre une déclaration d'ESSI pour sa contribution à l'innocuité des vaccins au Canada.

\section{Financement}

Ce travail a été entièrement financé par l'Agence de la santé publique du Canada.

\section{Références}

1. Huston P. Does Canada need to improve its immunization rates? Can Fam Physician 2017 Jan;63(1):e18. PubMed (https://www.ncbi.nlm.nih.gov/ pubmed/28115454?dopt=Abstract)

2. Governement du Canada. Taux de vaccination des enfants canadiens : faits saillants de l'Enquête sur la couverture vaccinale nationale des enfants. ASPC : 25 avril 2018. https://www.canada.ca/fr/sante-publique/services/ publications/vie-saine/2015-enquete-couverture-vaccinalenationale-enfants.html\#a1

3. Duclos P. La vaccinovigilance au Canada : Est-elle aussi robuste qu'elle le pourrait? Relevé des maladies transmissibles au Canada. 2014;40(Suppl 3):2-6. https:// www.canada.ca/fr/sante-publique/services/rapportspublications/releve-maladies-transmissibles-canada- 
rmtc/numero-mensuel/2014-40/rmtc-volume-40-s-3-4decembre-2014/rmtc-volume-40-s-3-4-decembre-2014-4. html

4. Agence de la santé publique du Canada. Système canadien de surveillance des effets secondaires suivant l'immunisation. ASPC : 9 décembre 2016. https://www.canada.ca/fr/ sante-publique/services/immunisation/systeme-canadiensurveillance-effets-secondaires-suivant-immunisation.html

5. Agence de la santé publique du Canada. Guide canadien d'immunisation: Partie 2-Innocuité des vaccins. ASPC: $1^{\mathrm{er}}$ septembre 2016. www.canada.ca/en/public-health/services/ publications/healthy-living/canadian- immunization-guidepart-2-vaccine-safety/page-2-vaccine-safety.html

6. Governement du Canada. Renseignements sur l'immunisation - provinces et territoires. Agence de la santé publique du Canada: 19 janvier 2018. https://www.canada. $\mathrm{ca} / \mathrm{fr} /$ sante-publique/services/renseignements-immunisationprovinces-et-territoires.html

7. Koch J, Leet C, McCarthy R, Carter A, Cuff W; Disease Surveillance Division, Bureau of Communicable Disease Epidemiology, Laboratory Centre for Disease Control. Adverse events temporally associated with immunizing agents: 1987 report. CMAJ 1989 Nov;141(9):933-7. PubMed (https://www.ncbi.nlm.nih.gov/ pubmed/2804852?dopt=Abstract)

8. Duclos P, McCarthy R, Koch J, Carter A; Bureau of Communicable Disease Epidemiology, Laboratory Centre for Disease Control. Effets secondaires reliés dans le temps à des agents immunisants (année 1988). Rapport hebdomadaire des maladies au Canada. 1990;16(32):157-66. PubMed (https://www.ncbi.nlm.nih.gov/ pubmed/2208346?dopt=Abstract)

9. Duclos P, Koch J, Hardy M, Carter A, McCarthy R; Bureau of Communicable Disease Epidemiology, Laboratory Centre for Disease Control. Effets secondaires reliés dans le temps à des agents immunisants (année 1989). Rapport hebdomadaire des maladies au Canada. 1991;17(29):147-52. PubMed (https://www.ncbi.nlm.nih.gov/ pubmed/1913934?dopt=Abstract)

10. Duclos P, Pless R, Koch J, Hardy M. Adverse events temporally associated with immunizing agents. Can Fam Physician 1993 Sep;39:1907-13. PubMed (https://www.ncbi. nlm.nih.gov/pubmed/8219839?dopt=Abstract)

11. Division de l'immunisation à l'enfance. Bureau de I'épidemiologie des maladies transmissibles (BEMT) au Laboratoire de lutte contre la maladie. Effets secondaires reliés dans le temps à des agents immunisants (année 1991). RMTC. 1993;19-20:168-179. http://publications.gc.ca/ collections/collection_2016/aspc-phac/HP3-1-19-20.pdf

12. Bentsi-Enchill A, Hardy M, Koch J, Duclos P. Childhood Immunization Division, Bureau of Communicable Disease Epidemiology, Laboratory Centre for Disease Control. AEffets secondaires reliés dans le temps à des vaccins - (rapport de 1992). Relevé des maladies transmissibles au Canada. PubMed (https://www.ncbi.nlm.nih.gov/ pubmed/7663460?dopt=Abstract)
13. Division of immunization, Bureau of Infectious Diseases, Laboratory Centre for Disease Control. Rapport sur I'immunisation au Canada, 1996. Chapitre 9. Surveillance des incidents indésirables associés au moment de l'administration du vaccin. Relevé des maladies transmissibles au Canada. 1997;23(Suppl 4):S24-7. http:// publications.gc.ca/collections/collection_2016/aspc-phac/ HP3-1-23-S4-eng.pdf

14. Canadian National Report on Immunization, 1997. Paediatr Child Health. 1998;3(Suppl B):25B-28B.

15. 1998 National Report (interim) on immunization Vaccine Safety Issues and Surveillance. Paediatr Child Health. 1999;4(Suppl C):26C-9C.

16. Agence de la santé publique du Canada. Rapport national sur l'immunisation au Canada, 2006. Relevé des maladies transmissibles au Canada. 2006;32(Suppl 3):1-44. http:// publications.gc.ca/collections/Collection/HP3-3-32S3E.pdf

17. Law BJ, Laflèche J, Ahmadipour N, Anyoti H. Rapport annuel du Système canadien de surveillance des effets secondaires suivant l'immunisation (SCSESSI) pour les vaccins administrés en 2012. Relevé des maladies transmissibles au Canada 2014;40(Suppl 3):S7- S21. https://www.canada.ca/fr/santepublique/services/rapports-publications/releve-maladiestransmissibles-canada-rmtc/numero-mensuel/2014-40/ rmtc-volume-40-s-3-4-decembre-2014/rmtc-volume-40-s-34-decembre-2014-5.html

18. Council for International Organizations of Medical Sciences (CIOMS) and World Health Organization. (WHO). Definition and Application of Terms for Vaccine Pharmacovigilance. Report of ClOMS/WHO Working Group on Vaccine Pharmacovigilance. Geneva, Switzerland: CIOMS and WHO; 2012. www.who.int/vaccine_safety/initiative/tools/CIOMS_ report_WG_vaccine.pdf

19. International Conference on Harmonisation (ICH) of Technical Requirements for Registration of Pharmaceuticals for Human Use. ICH Harmonised Tripartite Guideline. Clinical Safety Data Management: Definitions and Standards for Expedited reporting E2A. Current Step 4 version. ICH: Oct 27, 1994. www.ich.org/fileadmin/Public_Web_Site/ICH_ Products/Guidelines/Efficacy/E2A/Step4/E2A_Guideline.pdf

20. Agence de la santé publique du Canada. Déclaration de manifestations cliniques inhabituelles (mci) à la suite d'une immunisation. ASPC; Sept, 2016. https://www.canada.ca/ content/dam/phac-aspc/documents/services/immunization/ raefi-dmcisi-fra.pdf

21. Société canadienne de pédiatrie. Programme canadien de surveillance active de l'immunization (IMPACT). 2001-2016. https://www.cps.ca/fr/impact

22. Morris R, Halperin SA, Déry $P$, Mills E, Lebel M, MacDonald N, Gold R, Law BJ, Jadavji T, Scheifele D, Marchessault $V$, Duclos P. IMPACT monitoring network: A better mousetrap. Can J Infect Dis 1993 Jul;4(4):194-5. https://doi. org/10.1155/1993/262508. PubMed (https://www.ncbi.nlm. nih.gov/pubmed/22346446?dopt=Abstract)

23. Scheifele DW, Halperin SA; CPS/Health Canada, Immunization Monitoring Program, Active (IMPACT). Immunization Monitoring Program, Active: a model of 
active surveillance of vaccine safety. Semin Pediatr Infect Dis 2003 Jul;14(3):213-9. https://doi.org/10.1016/S10451870(03)00036-0. PubMed (https://www.ncbi.nlm.nih.gov/ pubmed/12913834?dopt=Abstract)

24. Groupe de travail sur la vaccinovigilance et l'Agence de la santé publique du Canada. Déclaration de manifestations cliniques inhabituelles $(\mathrm{MCl})$ à la suite d'une immunisation au Canada : Guide de l'utilisateur pour remplir et soumettre les rapports de déclaration de $\mathrm{MCl}$. Août 2011. https:// www.canada.ca/fr/sante-publique/services/immunisation/ declaration-manifestations-cliniques-inhabituelles-suiteimmunisation/guide-utilisateur-remplir-soumettre-rapportsdeclaration-mci.html

25. International Conference on Harmonisation (ICH). Support Documentation. Medical Dictionary for Regulatory Activities. www.meddra.org/how-to-use/supportdocumentation?current

26. SAS Enterprise Guide version 5.1. Cary, NC, USA: SAS Institute Inc., Copyright 2012. All Rights Reserved.

27. Statistique Canada. Estimations de la population au 1er juillet, par âge et sexe (Table 17-051-0001). CANSIM (base de données). https://www150.statcan.gc.ca/t1/tbl1/fr/tv.acti on?pid=1710000501\&request_locale $=f r$

28. Day A, Wang H, Quinn H, Cook J, Macarthy K. Annual report: surveillance of adverse events following immunisation in Australia, 2015. Commun Dis Intell 2017;41(3):E264-78. http://www.health.gov.au/internet/main/publishing.nsf/ Content/cdi4103-I

29. Harris T, Nair J, Fediurek J, Deeks SL. Assessment of sex-specific differences in adverse events following immunization reporting in Ontario, 2012-15. Vaccine 2017 May;35(19):2600-4. https://doi.org/10.1016/j. vaccine.2017.03.035. PubMed (https://www.ncbi.nlm.nih. gov/pubmed/28365252)

30. Agence de la santé publique du Canada. Couverture vaccinale des adultes canadiens : résultats de l'Enquête nationale sur la vaccination des adultes de 2014 (ENVA). ASPC: 2016. https://www.canada.ca/en/public-health/ services/publications/healthy-living/vaccine-uptakecanadian-adults-results-2014-adult-national-immunizationcoverage-survey.html

31. Zhou W, Pool V, Iskander JK, English-Bullard R, Ball R, Wise RP, Haber P, Pless RP, Mootrey G, Ellenberg SS, Braun MM, Chen RT. Surveillance for safety after immunization: Vaccine Adverse Event Reporting System (VAERS)-United States, 1991-2001. MMWR Surveill Summ 2003 Jan;52(1):1-24. PubMed (https://www.ncbi.nlm.nih.gov/ pubmed/12825543?dopt=Abstract)

32. Lawrence GL, Aratchige PE, Boyd I, Mclntyre PB, Gold MS. Annual report on surveillance of adverse events following immunisation in Australia, 2006. Commun Dis Intell Q Rep 2007 Sep;31(3):269-82. PubMed (https://www.ncbi.nlm.nih. gov/pubmed/17974219?dopt=Abstract)

33. Health Service (PHS). Food and Drug Administration (FDA) / Centers for Disease Control (CDC), Vaccine Adverse Event Reporting System (VAERS) 1990 - last month, CDC WONDER Online Database. https://wonder.cdc.gov/vaers. html

34. Bettinger JA, Halperin SA, Vaudry W, Law BJ, Scheifele DW; Le programme canadien de surveillance active de l'immunisation (IMPACT) : Surveillance active des effets secondaires suivant l'immunisation et des maladies évitables par la vaccination. Relevé des maladies transmissibles au Canada 2014;40(Suppl 3):41-4. PubMed (https://www.ncbi. nlm.nih.gov/pubmed/29769912?dopt=Abstract) 